\title{
Editorial: Inaugural issue
}

\author{
Juan Carlos Augusto ${ }^{\mathrm{a}}$ and Hamid Aghajan ${ }^{\mathrm{b}}$ \\ ${ }^{\text {a }}$ School of Computing and Mathematics and Computer Science Research Institute, University of Ulster, UK \\ ${ }^{\mathrm{b}}$ Department of Electrical Engineering, Stanford University, USA
}

\section{About the scientific area}

"Ambient Intelligence (AmI)" is a rapidly growing multi-disciplinary field allowing many areas of research to converge towards having a real beneficial influence in our society. The basic idea behind AmI is that by enriching an environment with technology (sensors, processors, actuators, information terminals, and other devices interconnected through a network), a system can be built such that based on the real-time information gathered and the historical data accumulated, decisions can be taken to benefit the users of that environment. AmI can be succinctly defined as "A digital environment that proactively, but sensibly, assists people in their daily lives" [2]. AmI is aligned with the concept of the disappearing computer: "The most profound technologies are those that disappear. They weave themselves into the fabric of everyday life until they are indistinguishable from it", wrote Mark Weiser, one of the founders of the ubiquitous computing field $[14,15]$.

Technology available today is rich. Several artifacts and items in a house can be enriched with sensors to gather information about their use and in some cases even to act independently without human intervention. Some examples of such devices are electro-domestic appliances (e.g., cooker and fridge), household items (e.g., taps, bed and sofa) and temperature handling devices (e.g., air conditioning and radiators). Expected benefits of this technology can be: (a) increasing safety (e.g., by monitoring lifestyle patterns or the latest activities and providing assistance when a possibly harmful situation is developing), (b) comfort (e.g., by adjusting temperature automatically), and (c) economy (e.g., controlling the use of lights). This abundance of technology has given place to the new notion of "Smart Environments (SmE)", which refers to environments that sense, perceive, interpret, project, react to, and anticipate the events of interest and offer services to users accordingly. Areas of application extend from homes to offices, shopping malls, factories, roadways, and to novel applications in multimedia and gaming.

Although Ambient Intelligence and Smart Environments are strongly related, we can distinguish them by going back to the old "mind/brain" metaphor used in Artificial Intelligence. While AmI is more concerned with the specific techniques to make an environment behave intelligently, SmE is more related to the intelligent interconnection of resources and their collective behavior. In other terms, while SmE aims to deal with the design of systems reactive and responsive to events, AmI also incorporates mechanisms for learning user preferences and behavior models over time. $\mathrm{AmI}$ and SmE share many common objectives and it is often difficult to separate them from each other. This is evident in the composition of topics in related conferences taking place around the world as these events attract interesting research in both areas. Such interconnection is reflected in the creation of JAISE, a journal addressing both areas.

One of the most popular instantiations of these areas is the concept of smart homes. Recent applications include the use of smart homes to provide a safe environment where people with special needs can have a better quality of life. For example, in the case of people at early stages of senile dementia (the most frequent case among the elderly suffering from Alzheimer's disease) the system can be tailored to minimize risks and ensure appropriate care at critical times by monitoring activities, diagnosing situations of interest and advising the carer. There are numerous ongoing academic research projects with well established smart homes research labs in this area, see for example Domus [13], MavHome [5], and Gator Tech Smart Home [8] in the US. The iDorm project [7] in the UK is another example, while many countries in the western half of Europe have smart home related programs (too many to list them all here). There are also many smart home 
programs in Asia. Three leading countries in the area are Japan, Singapore and Korea. Some examples of research and development programs in those countries are: WHT (the Welfare Techno Houses) [10] related to Chiba University (Japan), the Aware Home project [17] of NII (Japan), the Human-friendly Welfare Robot System Research Center [4] at KAIST (Korea) and the Institute for Infocomm Research (Singapore) [12]. Companies like Philips, Siemens, Nokia, Microsoft, SAP, VTT, and several European Telecoms are investing heavily in the areas of Ambient Intelligence and Smart Environments. Healthcare centres are also initiating their own smart home-based healthcare programs (for example Connected Health by the South Eastern Trust in Northern Ireland).

Although smart homes are currently the dominating force driving the SmE areas ahead many other research projects are based on different applications in smart offices, smart classrooms, and to increase safety for drivers in a car or employees in a production line. A summary of the potentials can be obtained from [3].

Above all we should not forget these systems were created to be immersed in a place where they will affect people's lives directly. Whether it is students in a classroom, people at home, pedestrians in a street or shoppers in a mall, their lives will be influenced by the technology deployed in those places. If the system works ideally, their life will be improved. If the system does not work as expected there is scope for disappointment [6,9].

Each environment should suit the particular needs of each person or group of people designated as the main beneficiaries of that environment. That means these systems are on the other side of the spectrum of "one size fit all". Obviously there is a core of technology and software that can be reused and it will be a mistake to build each application entirely from scratch. Still the adaptation of systems to each place and the combination, including possible conflicts amongst preferences of the primary users makes successful deployment a real challenge.

Privacy plays an important role on the acceptability of these systems. Take video cameras as an example. They can be used to monitor urban areas so that street crime can be detected as soon as possible. They can be used in smart homes to detect situations where somebody may be at risk $[1,11,16]$, which is an extremely valuable safety net for anyone who is in a vulnerable situation. But the very same reason that justifies the use of cameras can be also a reason for some to reject them: the big brother scenario and the fear of the loss of privacy. Depending on the benefits expected from the system, some users may be willing to give up some degree of privacy in return for increased safety.

Behind the perception of the loss of privacy is a fear that people other than those strictly authorized to access private information can use it in a negative way. Users want to see that security is an integral part of the system and that information will not be accessed by anyone who can use it for the wrong reasons. Handling these types of problems is often beyond the comfort zone of many researchers devoted to algorithm design, while the proper addressing of issues such as privacy and security is key to gaining user acceptance for many SmE and AmI applications.

\section{About the journal}

There are growing indications that the topics of Ambient Intelligence and Smart Environments will reshape the world as we know it today. Due to the importance of the subject and its inter-disciplinary nature, a significant amount of research is advancing the area in different fronts. The underpinning conceptual frameworks, the technology, and thus the research world is ready for a global, multi-disciplinary, and broad community to shape up and bring about applications based on AmI-SmE. Hence, we believe JAISE is timely and offers a unique forum for this growing community.

JAISE aims to promote participation from several different communities covering topics ranging from enabling technologies such as multi-modal sensing and vision processing, to algorithmic aspects in interpretive and reasoning domains, to application-oriented efforts in human-centered services, as well as contributions from the fields of robotics, networking, HCI, mobile, collaborative, and pervasive computing. This diversity stems from the fact that smart environments can be defined with a variety of different characteristics based on the applications they serve, their interaction models with humans, the practical system design aspects, as well as the multi-faceted conceptual and algorithmic considerations that would enable them to operate seamlessly and unobtrusively. As such, societal implications of the new applications based on sensing human activities as well as the psychological and user acceptance studies of the technology adoption are also included as areas of relevance and interest.

Reflecting the multi-disciplinary character of this area the journal will feature Thematic Issues which will highlight the importance of the specific topic. 
Two such Thematic Issues have been planned for the first volume. One will be focused on the importance and role of sensing/actuating devices as a fundamental technology for the area. Another Thematic Issue will consider the role of Artificial Intelligence in creating autonomous environments and sensitive systems that can serve human beings.

We hope the different communities participating in the development of Ambient Intelligence and Smart Environments will share our vision to identify JAISE as a medium for the exchange of the most innovative results and the latest research trends in the various topics involved in AmI and SmE. We further hope that JAISE will provide opportunities for researchers across the different related fields to learn about ongoing work in other areas and explore potentials of multi-disciplinary cooperation.

\section{About this inaugural issue}

We are very pleased to announce the first issue of what we hope will be a standing place for publishing AmI and SmE research. This first issue consists of invited articles from leading researchers in the area. The articles provide critical surveys of important sub-areas which have a strategic value for the community. These articles also provide a vision of the evolution of such areas and an agenda for research.

In "New Research Perspectives on Ambient Intelligence", Emile Aarts and Boris de Ruyter offer a number of new AmI research perspectives that are related to social intelligence, and introduce the concept of Experience Research aimed at developing methods that allow the validated feedback of users as the center of the interaction.

Sensing and interpretation of human activities is a gateway to understanding actions and intentions of users for offering services and creating virtual representations. In "Sensory Grammars for Sensor Networks", Yannis Aloimonos suggests that human activity can be expressed in a language which can be learned using machine learning techniques applied to sensed data from the user. The paper argues that such a language can act as a bridge between the different areas involved in the interpretation of human action.

Much of the high-level recognition of user's explicit and implicit input to an HCI system is based on features extracted from multiple sensing modalities. Human pose, speech, gesture, gaze, movement patterns, and other behavioral cues are often acquired through disjoint systems. In "Multimodal Interfaces: Challenges and Perspectives", Nicu Sebe reviews the major approaches to multi-modal HCI in human-centric applications, and discusses techniques for user and task modeling via multimodal fusion.

Spoken dialogue is a key enabler of user-centric human-computer interaction. In "The Role of Spoken Language Dialogue Interaction in Intelligent Environments”, Wolfgang Minker, Ramón López-Cózar, and Michael McTear outline the related research areas and discuss future trends in assistive, adaptive and proactive system design, dialogue management and systemenvironment interaction.

Touch is an alternative and complementary channel for communication between users and the system. In "Haptic Interaction Becomes Reality", Roope Raisamo, Veikko Surakka, Jukka Raisamo, Jussi Rantala, Jani Lylykangas, and Katri Salminen offer an introduction to the state of the art in haptic interaction technology and its promises in interactive systems, as well as in communication between humans.

Developing large-scale experiments involving numerous sensing methods and a large number of human participants creates challenges in experiment design. In "Participatory Technologies for Designing Ambient Intelligence Systems", Toru Ishida and Hiromitsu Hattori discuss participatory simulations in which multiagents include human-controlled avatars and scenarioguided agents. They also discuss augmented experiments which enable performing real-world large-scale trials with a small number of human subjects.

In "Multi-Agent Smart Environments", Diane Cook discusses the role of multi-agent research in the context of Smart Environments and offers a survey of current research in the area. The article also poses several challenges in this dynamic and complex area of research.

In "Modeling and Intelligibility In Ambient Environments", Anind Dey introduces the notion of intelligibility, or the ability of applications to explain their behavior to the user. As an application feature, intelligibility includes supporting users in understanding why the system takes a particular action in response to a sensory input.

In "Second Order Ambient Intelligence", Marc Böhlen imagines a future in which the current issues and challenges within Ambient Intelligence have been resolved, and formulates a set of new topics and ideas that can be part of a so-termed second order Ambient Intelligence. 
Finally, in "Soft-Appliances: A Vision for User Created Networked Appliances in Digital Homes", Jeannette Chin, Vic Callaghan, and Graham Clarke describe a vision for a new type of domestic appliance, a soft-appliance, constructed from the aggregation of elementary network services. The metaappliance/application concept is proposed as a semantic data template that describes the soft or virtual appliance that can be instantiated by manufactures or endusers in a way that redefines the nature of an appliance.

\section{References}

[1] H. Aghajan, J.C. Augusto, C. Wu, P. McCullagh, and J.-A. Walkden. Distributed vision-based accident management for assisted living. In T. Okadome, T. Yamazaki, and M. Mokhtari, editors, In "Pervasive Computing for Quality of Life Enhancement", Proceedings of the 5th International Conference On Smart Homes and Health Telematics. Nara, Japan, volume 4541 of Lecture Notes in Computer Science, pages 196-206, 2007.

[2] J.C. Augusto. Ambient Intelligence: the Confluence of Ubiquitous/Pervasive Computing and Artificial Intelligence, pages 213-234. Intelligent Computing Everywhere. Springer London, 2007.

[3] J.C. Augusto and D.J. Cook. Ambient Intelligence: applications in society and opportunities for AI. 20th International Joint Conference on Artificial Intelligence (IJCAI-07). 2007.

[4] Z.Z. Bien, H.-E. Lee, J.-H. Do, Y.-H. Kim, K.-H. Park, and S.-E. Yang. Intelligent interaction for human-friendly service robot in smart house environment. International Journal of Computational Intelligence System, 1(1):77-93, 2008.

[5] S.K. Das, D.J. Cook, A. Bhattacharya, E.O. Heierman, and T.Y. Lin. The role of prediction algorithms in the mavhome smart home architecture. IEEE Wireless Communications, 9(6):7784, 2002.
[6] M. Dertouzos. Human-centered systems. In P. Denning, editor, The Invisible Future, pages 181-192, 2001.

[7] F. Doctor, H. Hagras, and V. Callaghan. A fuzzy embedded agent-based approach for realizing ambient intelligence in intelligent inhabited environments. IEEE Transactions on Systems, Man, and Cybernetics, Part A, 35(1):55-65, 2005.

[8] A. Helal, W. Mann, H. Elzabadani, J. King, Y. Kaddourah, and E. Jansen. Gator tech smart house: A programmable pervasive space. IEEE Computer magazine, pages 64-74, March 2005.

[9] P. Huuskonen. Run to the hills! ubiquitous computing meltdown. In J.C. Augusto and D. Shapiro, editors, Advances in Ambient Intelligence, volume 164 of Frontiers in Artificial Intelligence and Applications (FAIA), pages 157-172. IOS Press, 2007.

[10] A. Kawaradal, T. Takagi, A. Tsukadal, K. Sasakil, M. Ishijima, T. Tamura, T. Togawa, and K. Yamakoshi. Evaluation of automated health monitoring system at the "welfare techno house". In Proceedings of the 20th Annual International Conference of the IEEE Engineering in Medicine and Biology Society, volume 20, 1998.

[11] A. Keshavarz, A.M. Tabar, and H. Aghajan. Distributed visionbased reasoning for smart home care. In In Proc. of ACM SenSys Workshop on DSC, 2006.

[12] B.Y. Lim, D. Zhang, M. Zhu, and S. Zheng. Context-aware framework for spontaneous interaction of services in multiple heterogeneous spaces. In ICME, pages 328-331, 2007.

[13] H. Pigot, A. Mayers, S. Giroux, B. Lefebvre, and N. Noury V. Rialle. Smart house for frail and cognitive impaired elders. September 2002.

[14] M. Weiser. The computer for the twenty-first century. Scientific American, 165:94-104, 1991.

[15] M. Weiser. Hot topics: Ubiquitous computing. IEEE Computer, 26(10):71-72, 1993.

[16] C. Wu, H. Aghajan, and R.P. Kleihorst. Real-time human posture reconstruction in wireless smart camera networks. In Proceedings of IPSN'08, pages 321-331, 2008.

[17] T. Yamazaki, K. Kayama, and S. Igi. Detection of people and tracking test bed using multiple sensors. In MUE, pages 135140, 2007. 